\title{
Extinction Efficiency and Electromagnetic Fields of Isolated and Coupled Core-Shell Nanoparticles
}

\author{
André F. S. da Cruz and Karlo Q. da Costa
}

\begin{abstract}
The study of metallic nanoparticles fed by optical fields has great interest in nanophotonics, for example in sensing devices. This paper presents a theoretical study of the interaction between electromagnetic waves and gold nanostructures with spherical geometries, which have a thin dielectric layer of silica. It is considered that the particle's size is much smaller than the operating wavelength, characterizing the Rayleigh scattering regime. Using an analytical model through the Laplace equation, the interaction between an oscillating uniform electric field and a core-shell nanosphere is presented. Then, using a numerical model, the scattering of two interacting core-shell nanospheres is also analyzed, as a function of the distance between them. For the isolated particle case, the efficiency parameters of scattering, absorption and extinction cross sections were calculated and compared with experimental data of absorbance curves. The results were obtained in the range of wavelengths from $450 \mathrm{~nm}$ to $750 \mathrm{~nm}$. Some conclusions about the range of validity of the model in functions of the particle's dimensions are presented.
\end{abstract}

Index Terms-Rayleigh Scattering, Gold Nanoparticles, CoreShell, Analytical Modeling, Finite Element Method.

\section{INTRODUCTION}

C URRENTLY, the study of metallic nanoparticles and their optical response has been of great interest in different areas, such as biochemistry, engineering and nanomedicine, with applications, for example, in cancer treatments and diagnostics through bio-imaging, phototherapy and circulating biomarkers in blood fluids [1,2], as well as the design of photonic sensing structures for chemical process analyzes [3], this is mainly due to its plasmonic effects [4].

Several techniques for the production of metallic nanoparticles (NPM) have made it possible to obtain different sizes and geometric shapes, from cylinders to triangular shapes, in addition to spherical shape approximations. Recently, chemical reactions in liquid solutions have been commonly used as a low cost procedure for NPM production. From this process, the presence of the surfactant agent, in general, a polymer that binds to the surface of the nanoparticle during the chemical reaction, controlling the growth rate and providing stability to the sample [5-6] is highlighted. The result is a type of metal particle covered with a thin superficial dielectric layer, which is called core-shell.

In this method of manufacture the dispersion, or variation, of size and shape of the nanoparticles in a sample is common. This dispersion makes it difficult to interpret characterization and identification measures, since its optical properties are strongly related to the NPM dimensions, besides the intrinsic

A. F. S. Cruz is with Federal University of Pará (UFPA), Campus Guamá, Brazil (email: felipe.andcruz@gmail.com).

K. Q. Costa is with Federal University of Pará (UFPA), Campus Tucuruí, Brazil (email: karlo@ufpa.br). characteristics of the medium where they are included. In this context, it's important to study the effect of small variations in particle size and core-shell on their resonant optical responses, in order to obtain more precise theoretical results.

The theoretical study of electromagnetic scattering can offer satisfactory results on the sensing of these NPMs [5]. The interaction between metals and waves in the optical range produces an oscillatory behavior of the free electrons cloud at the opposite phase of the incident field, such an effect is directly related to the negative real part of the complex metal dielectric function. The result of this interaction is known as plasmon wave [7]. Plasmonic effects on metals suggest differences between microwave (RF) and optical wave regimes, since, in the optical regime, metals are considered to be imperfect conductors [8].

Recently, gold nanoparticles used as molecular optical probes have shown wide application and great prospects; this is due to the resonant plasmon characteristic of gold. These studies have contributed significantly to the development of biosensors, molecular images and photothermal treatments of diseases $[1,9]$. Gold nanoparticles, which are excited by laser source of wavelength close to the SPR band, can efficiently convert photon energy into thermal energy, leading to the destruction of biological cells such as tumors and bacteria $[9,10]$. When a sample of material, solid or liquid, contains a dispersion of nanometric particles, the medium is defined as colloid [5]. The absorbance is a parameter that associates the extinction coefficient of a set of nanoparticles with the characteristics of the colloid, this parameter is related to the phenomenon of localized surface plasmon resonance. The SPR absorption band analysis can provide information on the dimensions and structure of the metal nanoparticle. The geometric and constituent characteristics of the particles and the medium directly influence the plasmon resonance bandwidth [9.10]. The presence of the dielectric layer changes the spectral position, and in this way, it must be considered in the mathematical models, making the analysis more precise in the development of optical equipment.

The interaction between a particle of effective radius $r$ and an optical beam can be analyzed using a quasi-static approximation, provided that the particle's dimensions are much smaller than the wavelength of the field in focus, i.e. $r \ll \lambda$, in this case the phase of harmonic electromagnetic field is practically constant nearly the particle, besides making possible the calculation of the total space field distribution by a simplified electrostatic problem [8]. This method is known as quasi-static problem or Rayleigh scattering.

In this work an analytical study of the interaction between a core-shell type particle, having a spherical core of gold 
covered by a layer of silica, and an electromagnetic field using the quasi-static model of Rayleigh, which is an approximation of the Mie scattering. The objective is to analyze the total field, in the internal and external regions of the NPM, and to carry out a parametric analyzes of the transversal sections of scattering, absorption and extinction. Also, we investigate the effects of the presence and absence of a layer dielectric, and compare the absorbance data obtained analytically with experimental data available in [12]. A second analysis is presented to verify the electromagnetic coupling between two core-shell NPMs. For this case, a software based on the finite element method was used [11].

\section{Analytical Modeling}

A. Description of the Problem and General Solution of the Laplace Equation in Spherical Coordinates with Azimuthal Symmetry

The core-shell geometry is characterized by two concentric spheres at the origin, where the inner sphere has a radius $r_{1}$ and the outer radius $r_{2}$ (Fig. 1). This particle is constituted by an internal core with a dielectric constant $\varepsilon_{1}$ coated by a layer of thickness $\Delta=r_{2}-r_{1}$ with dielectric constant $\varepsilon_{2}$, where both materials are homogeneous and isotropic. The particle is immersed in an external medium with permittivity $\varepsilon_{m}$, and a plane wave illuminates it in the quasi-static regime. This plane wave produces a oscillating uniform electric field that interact with the particle.

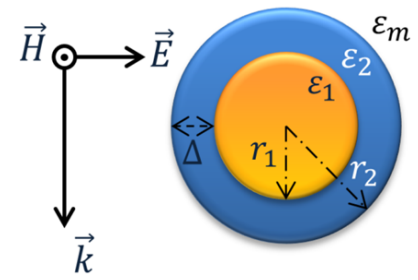

Fig. 1. Core-Shell particle in the presence of an oscillating uniform field.

To determine the total electric field resulting from the interaction between the plane wave and core-shell particle, it is necessary to find the solution of the Laplace boundary value problem (BVP) given by (1). This differential equation of potential is in terms of spatial variables, and the harmonic dependence with temporal variable can be introduced in the final solution as suggested by the Rayleigh method.

$$
\frac{1}{r^{2}} \frac{\partial}{\partial r}\left(r^{2} \frac{\partial V}{\partial r}\right)+\frac{1}{r^{2} \sin \theta} \frac{\partial}{\partial \theta}\left(\sin \theta \frac{\partial V}{\partial \theta}\right)+\frac{1}{r^{2} \sin ^{2} \theta} \frac{\partial^{2} V}{\partial \phi^{2}}=0
$$

We can solve this partial differential equation (PDE) using the method of separation of variables. This is a well-known technique in the literature, where the solution of (1) is given in the form of a product solution $V(r, \theta, \phi)=R(r) \Theta(\theta) \Phi(\phi)$ obtained from three ordinary differential equations (ODE). For the proposed problem, the ordinary differential equations are:

$$
\begin{gathered}
\frac{\partial^{2} \Phi}{\partial \phi^{2}}+m^{2} \Phi=0 \\
\frac{\partial}{\partial r}\left(r^{2} \frac{\partial R}{\partial r}\right)-\gamma R=0 \\
\frac{1}{\sin \theta} \frac{\partial}{\partial \theta}\left(\sin \theta \frac{\partial \Theta}{\partial \theta}\right)+\Theta\left(\gamma-\frac{m^{2}}{\sin ^{2} \theta}\right)=0
\end{gathered}
$$

where (3) and (4) are known as differential equations of Cauchy-Euler and Legendre, respectively [18], with $m$ and $\gamma$ being the separation constants.

For problems with azimuth symmetry, the electric potential does not present variation with $\phi$ coordinate, therefore $m=0$, reducing the solution of (2) to an arbitrary constant. Equations (3) and (4) can be solved from the Frobenius series method and for (3), due to the finiteness condition in the origin, the solution is converted into Legendre polynomials of the first type $[19,20]$.

A stronger consideration for this solution is that a combination of Legendre polynomials may better satisfies the boundary conditions that the spherical problem may present [21], so the general solution obtained is:

$$
V(r, \theta)=\sum_{n=0}^{\infty}\left(a_{n} r^{n}+b_{n} r^{-(n+1)}\right) P_{n}(\cos \theta)
$$

\section{B. Solution of the Rayleigh Problem for a Core-Shell Particle}

The electric field must exist in three regions: in the core, in the region of the dielectric layer and in the external medium to the particle, each with the potential defined by:

$$
\begin{aligned}
& V_{1}(r, \theta)=\sum_{n=0}^{\infty}\left(a_{n} r^{n}+b_{n} r^{-(n+1)}\right) P_{n}(\cos \theta): r<r_{1} \\
& V_{2}(r, \theta)=\sum_{n=0}^{\infty}\left(c_{n} r^{n}+d_{n} r^{-(n+1)}\right) P_{n}(\cos \theta): r_{1} \leq r<r_{2} \\
& V_{3}(r, \theta)=\sum_{n=0}^{\infty}\left(e_{n} r^{n}+f_{n} r^{-(n+1)}\right) P_{n}(\cos \theta): r \geq r_{2}
\end{aligned}
$$

It is observed that the (6) has singularity at the origin, which is undesirable, however, such problem can easily be solved by doing $b_{n}=0$. The constants in (6)-(8) are obtained by applying the following boundary conditions at the boundaries $r=r_{1}, r=r_{2}$ and $r \rightarrow \infty$ :

$$
\begin{aligned}
&-\varepsilon_{1}{\frac{\partial V_{1}}{\partial r}}_{r=r_{1}}=-\varepsilon_{2}{\frac{\partial V_{2}}{\partial r}}_{r=r_{1}} \\
&-\frac{1}{r}{\frac{\partial V_{1}}{\partial \theta}}_{r=r_{1}}=-\frac{1}{r}{\frac{\partial V_{2}}{\partial \theta}}_{r=r_{1}} \\
&-\varepsilon_{2}{\frac{\partial V_{2}}{\partial r}}_{r=r_{2}}=-\varepsilon_{m} \frac{\partial V_{3}}{\partial r}{ }_{r=r_{2}} \\
&-\frac{1}{r}{\frac{\partial V_{2}}{\partial \theta}}_{r=r_{2}}=-\frac{1}{r} \frac{\partial V_{3}}{\partial \theta}{ }_{r=r_{2}} \\
& \lim _{r \rightarrow \infty}-\nabla V_{3}=E_{o} \widehat{a}_{z}
\end{aligned}
$$


where $E_{0}$ is the electric field of the plane wave. Substituting (6)-(8) into (9)-(13), it is found that the conditions are satisfied only for $n=1$, so the constants are obtained:

$$
\begin{aligned}
& a_{1}=-3 \varepsilon_{m} \frac{3 \varepsilon_{2}}{f\left(\varepsilon_{1}-\varepsilon_{2}\right)\left(2 \varepsilon_{2}-2 \varepsilon_{m}\right)+\left(2 \varepsilon_{2}+\varepsilon_{1}\right)\left(\varepsilon_{2}+2 \varepsilon_{m}\right)} E_{0} \\
& c_{1}=-3 \varepsilon_{m} \frac{\left(2 \varepsilon_{2}+\varepsilon_{1}\right)}{f\left(\varepsilon_{1}-\varepsilon_{2}\right)\left(2 \varepsilon_{2}-2 \varepsilon_{m}\right)+\left(2 \varepsilon_{2}+\varepsilon_{1}\right)\left(\varepsilon_{2}+2 \varepsilon_{m}\right)} E_{0} \\
& d_{1}=r_{1}^{3} \frac{3 \varepsilon_{m}\left(\varepsilon_{1}-\varepsilon_{2}\right)}{f\left(\varepsilon_{1}-\varepsilon_{2}\right)\left(2 \varepsilon_{2}-2 \varepsilon_{m}\right)+\left(2 \varepsilon_{2}+\varepsilon_{1}\right)\left(\varepsilon_{2}+2 \varepsilon_{m}\right)} E_{0} \\
& f_{1}=r_{2}^{3}\left(\frac{f\left(\varepsilon_{1}-\varepsilon_{2}\right)\left(2 \varepsilon_{2}+\varepsilon_{m}\right)+\left(2 \varepsilon_{2}+\varepsilon_{1}\right)\left(\varepsilon_{2}-\varepsilon_{m}\right)}{f\left(\varepsilon_{1}-\varepsilon_{2}\right)\left(2 \varepsilon_{2}-2 \varepsilon_{m}\right)+\left(2 \varepsilon_{2}+\varepsilon_{1}\right)\left(\varepsilon_{2}+2 \varepsilon_{m}\right)}\right) E_{0}
\end{aligned}
$$

where $f=r_{1}^{3} / r_{2}^{3}$ is the fraction of the total volume of the particle occupied by the inner sphere.

Thus, the electric potential is determined in the regions:

$$
V(r, \theta)= \begin{cases}-\frac{3 \varepsilon_{2}}{\zeta} E_{0} r \cos \theta & : r<r_{1} \\ -\frac{\left(2 \varepsilon_{2}+\varepsilon_{1}\right)}{\zeta} E_{0} r \cos \theta+\frac{p_{\text {in }}}{4 \pi \varepsilon_{2} r^{2}} \cos \theta & : r_{1} \leq r<r_{2} \\ -E_{0} r \cos \theta+\frac{p_{\text {out }}}{4 \pi \varepsilon_{m} r^{2}} \cos \theta & : r \geq r_{2}\end{cases}
$$

being $\zeta=\frac{f\left(\varepsilon_{1}-\varepsilon_{2}\right)\left(2 \varepsilon_{2}-2 \varepsilon_{m}\right)+\left(2 \varepsilon_{2}+\varepsilon_{1}\right)\left(\varepsilon_{2}+2 \varepsilon_{m}\right)}{3 \varepsilon_{m}}$.

An analysis of the electric potential in the regions of the particle reveals the appearance of a characteristic behavior known in the electrostatic, the dipole, so it is useful to define the term polarizability, which is directly related to the amplitude of the induced dipole moment. The internal and external dipole moments are defined by:

$$
\begin{gathered}
p_{\text {in }}=\alpha_{\text {in }} E_{0} \\
p_{\text {out }}=\alpha_{\text {out }} E_{0}
\end{gathered}
$$

where the internal and external polarizabilities are:

$$
\begin{gathered}
\alpha_{\text {in }}=4 \pi \varepsilon_{2} r_{1}^{3} \frac{3 \varepsilon_{m}\left(\varepsilon_{1}-\varepsilon_{2}\right)}{f\left(\varepsilon_{1}-\varepsilon_{2}\right)\left(2 \varepsilon_{2}-2 \varepsilon_{m}\right)+\left(2 \varepsilon_{2}+\varepsilon_{1}\right)\left(\varepsilon_{2}+2 \varepsilon_{m}\right)} E_{0} \\
\alpha_{\text {out }}=4 \pi \varepsilon_{m} r_{2}^{3}\left(\frac{f\left(\varepsilon_{1}-\varepsilon_{2}\right)\left(2 \varepsilon_{2}+\varepsilon_{m}\right)+\left(2 \varepsilon_{2}+\varepsilon_{1}\right)\left(\varepsilon_{2}-\varepsilon_{m}\right)}{f\left(\varepsilon_{1}-\varepsilon_{2}\right)\left(2 \varepsilon_{2}-2 \varepsilon_{m}\right)+\left(2 \varepsilon_{2}+\varepsilon_{1}\right)\left(\varepsilon_{2}+2 \varepsilon_{m}\right)}\right) E_{0}
\end{gathered}
$$

Applying the gradient operation in spherical coordinates to (19) and adding the temporal harmonic variation, we find the electric field in the all regions:

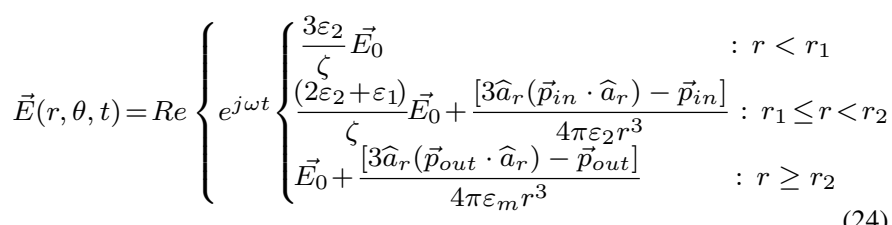

where $\widehat{a}_{r}$ is the unit vector normal to the surface of the sphere.
From (24) it's verified that the total field outside the particle $\left(r>r_{2}\right)$ can be represented by the sum of the radiation of an equivalent hertzian dipole and the incident plane wave field $E_{0}$. From this equation, we obtain through the Maxwell equations the field $H(t)$ harmonically oscillating in the vicinity of the particle $[8,22,23]$ :

$$
\vec{H}(r, \theta, t)=\operatorname{Re}\left\{e ^ { j \omega t } \left\{\begin{array}{cc}
0 & : r<r_{1} \\
j \omega \frac{1}{4 \pi r^{2}}\left(\widehat{a}_{r} \times \vec{p}_{\text {in }}\right) & : r_{1} \leq r<r_{2} \\
j \omega \frac{1}{4 \pi r^{2}}\left(\widehat{a}_{r} \times \vec{p}_{\text {out }}\right) & : r \geq r_{2}
\end{array}\right.\right.
$$

Note that the magnetic field has no component in the direction $\widehat{a}_{z}$.

\section{Efficiency of Scattering, Absorption and Extinction}

The cross-sections of scattering and absorption are defined, respectively, by [5]:

$$
\begin{aligned}
C_{\text {sca }} & =\frac{k^{4}}{6 \pi}\left|\alpha_{\text {out }}\right|^{2} \\
C_{\text {abs }} & =k \operatorname{Im}\left[\alpha_{\text {out }}\right]
\end{aligned}
$$

being $\alpha_{\text {out }}$ the equivalent polarizability of the particle.

The extinction cross section is defined by the ratio of the extinction of light power through absorption and scattering to incident light intensity $[8,15]$. In equation form we have:

$$
C_{e x t}=C_{s c a}+C_{a b s}
$$

The dispersion efficiency of scattering, absorption or extinction (total), is defined as the cross section of (26)-(27) normalized to the sectional area of the particle $(S)$.

$$
\begin{aligned}
Q_{s c a} & =\frac{C_{s c a}}{S} \\
Q_{a b s} & =\frac{C_{a b s}}{S}
\end{aligned}
$$

\section{Relative Permittivity of Materials}

The core of the nanoparticle is composed of gold, and its complex permissiveness is defined by the Lorentz-Drude model, which considers the effects of interband and intraband [13]. However, the reduced size of the nanoparticles limits the average path of the free electrons, resulting in a considerable increase of the scattering rate. Thus, it is necessary to introduce a dependence on the radius of the NPM, which represents in an approximate way this phenomenon [5]. The permissiveness of gold by the model used is:

$$
\varepsilon_{\text {gold }}(\omega)=\varepsilon_{\infty}-\frac{\omega_{p 1}^{2}}{\omega^{2}+j\left(\Gamma+B \frac{\nu_{m}}{R}\right) \omega}+\frac{\omega_{p 2}^{2}}{\omega_{0}^{2}-\omega^{2}-j \gamma \omega}
$$

where $\varepsilon_{\infty}$ is the relative permittivity for infinite frequency, $\omega_{p 1}$ and $\omega_{p 1}$ are the plasma frequencies, $\gamma$ and $\Gamma$ are the damping frequencies, $\nu_{m}$ is the Fermi velocity, $\omega_{0}=2 \pi c / \lambda_{0}$ is the angular frequency for the wavelength specific $\lambda_{0}=$ 
$450 \mathrm{~nm}, R$ is the radius of the NPM and $B$ is a stabilizing constant of model experimentally defined [5,13].

We can verify the behavior of the dielectric function by varying in frequency with the change of particle radius (Fig. 2).
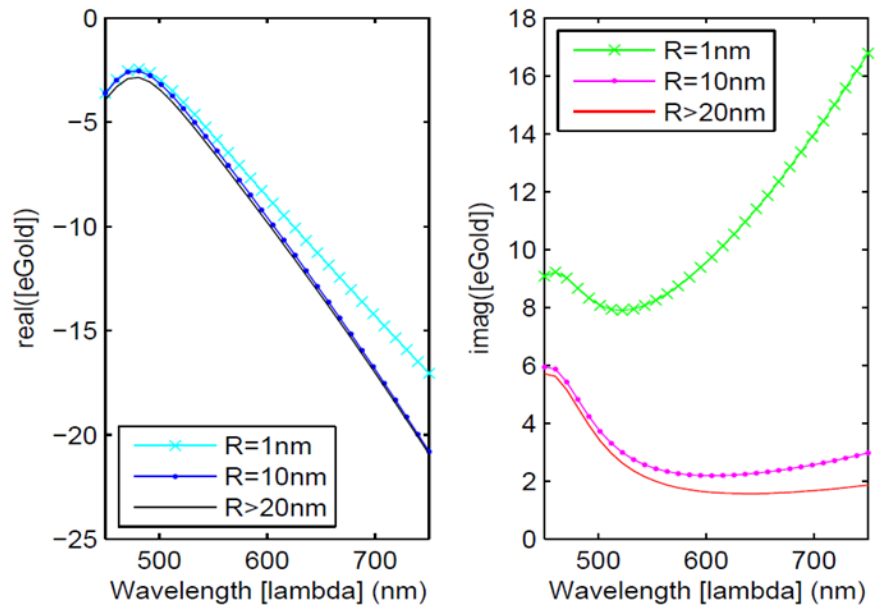

Fig. 2. Relative Permittivity for three radius (a) Real Part, (b) Imaginary Part

The stabilizing layer covering the nanoparticle is silica, which has relative permittivity defined by the Sellmeier model. This is an empirical relationship between the index of refraction and the wavelength for a particular transparent medium [14]:

$$
\varepsilon_{\text {silica }}(\lambda)=1+\frac{B_{1} \lambda^{2}}{\lambda^{2}-C_{1}}+\frac{B_{2} \lambda^{2}}{\lambda^{2}-C_{2}}+\frac{B_{3} \lambda^{2}}{\lambda^{2}-C_{3}}
$$

where $C_{1}, C_{2}, C_{3}, B_{1}, B_{2}$, and $B_{3}$ are the Sellmeier coefficients determined experimentally in [16]. The curve for the model is shown in Fig. 3.

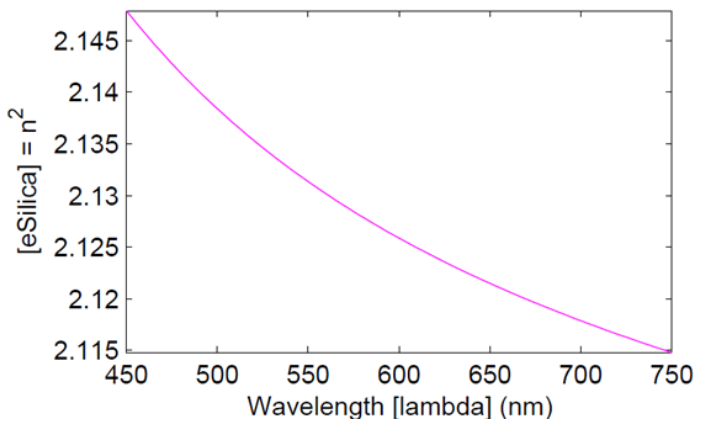

Fig. 3. Relative permittivity of silica.

The permittivity of the medium was similarly defined by the Sellmeier model using the coefficients defined in [16], these consider that the layer and the medium are non-absorbent, having only real part in the dielectric function.

Values used in (31) and (32) are shown in Table I, all according to SI:
TABLE I

VALUES USED IN (31) AND (32)

\begin{tabular}{|c|c|c|}
\hline Symbol & Quantity & Value \\
\hline$\varepsilon_{\infty}$ & $\begin{array}{l}\text { Relative permittivity } \\
\text { for infinite frequency }\end{array}$ & 7 \\
\hline$\omega_{p 1}$ & Plasma frequency 1 & $1.3579 \times 10^{16} \mathrm{rad} / \mathrm{s}$ \\
\hline$\omega_{p 2}$ & Plasma frequency 2 & $4.4910 \times 10^{15} \mathrm{rad} / \mathrm{s}$ \\
\hline$\omega_{0}$ & $\begin{array}{l}\text { Angular frequency for } \\
\lambda_{0}=450 \mathrm{~nm}\end{array}$ & $4.1888 \times 10^{15} \mathrm{rad} / \mathrm{s}$ \\
\hline$\Gamma$ & $\begin{array}{l}\text { Damping frequency - } \\
\text { interbanda }\end{array}$ & $9.9909 \times 10^{13} \mathrm{rad} / \mathrm{s}$ \\
\hline $4 \gamma$ & $\begin{array}{l}\text { Damping frequency - } \\
\text { intrabanda }\end{array}$ & $8.9516 \times 10^{14} \mathrm{rad} / \mathrm{s}$ \\
\hline$\nu_{m}$ & Fermi velocity for gold & $1.4 \times 10^{16} \mathrm{~m} / \mathrm{s}$ \\
\hline$B$ & $\begin{array}{l}\text { Stabilizing constant of } \\
\text { model experimentally } \\
\text { defined }[2,10] \\
\text { Complex number }\end{array}$ & $(0.85+0.35 j) \mathrm{rad}$ \\
\hline$B_{1}$ & $\begin{array}{l}\text { Sellmeier coefficients } \\
\text { for silica }\end{array}$ & 0.6961663 \\
\hline$B_{2}$ & $\begin{array}{l}\text { Sellmeier coefficients } \\
\text { for silica }\end{array}$ & 0.4079426 \\
\hline$B_{3}$ & $\begin{array}{l}\text { Sellmeier coefficients } \\
\text { for silica }\end{array}$ & 0.8974794 \\
\hline$C_{1}$ & $\begin{array}{l}\text { Sellmeier coefficients } \\
\text { for silica }\end{array}$ & $0.068404^{2} \times 10^{12}$ \\
\hline$C_{2}$ & $\begin{array}{l}\text { Sellmeier coefficients } \\
\text { for silica }\end{array}$ & $0.116241^{2} \times 10^{12}$ \\
\hline$C_{3}$ & $\begin{array}{l}\text { Sellmeier coefficients } \\
\text { for silica }\end{array}$ & $9.896161^{2} \times 10^{12}$ \\
\hline
\end{tabular}

\section{NumericAl MODELING}

In real sample of particles there are large numbers of NPMs distributed randomly, which a mutually interacting with each other. In the case of low density of NPMs, the interactions between particles can be reduced to an isolated particle. But if the NPM density is high, the interaction between them must be considered. In these cases, a numerical method based in Finite Element Method (FEM) with periodicity boundary condition can be used to analyze a set of two NPMs separated by a distance $z_{\Delta}$ (Fig. 4).

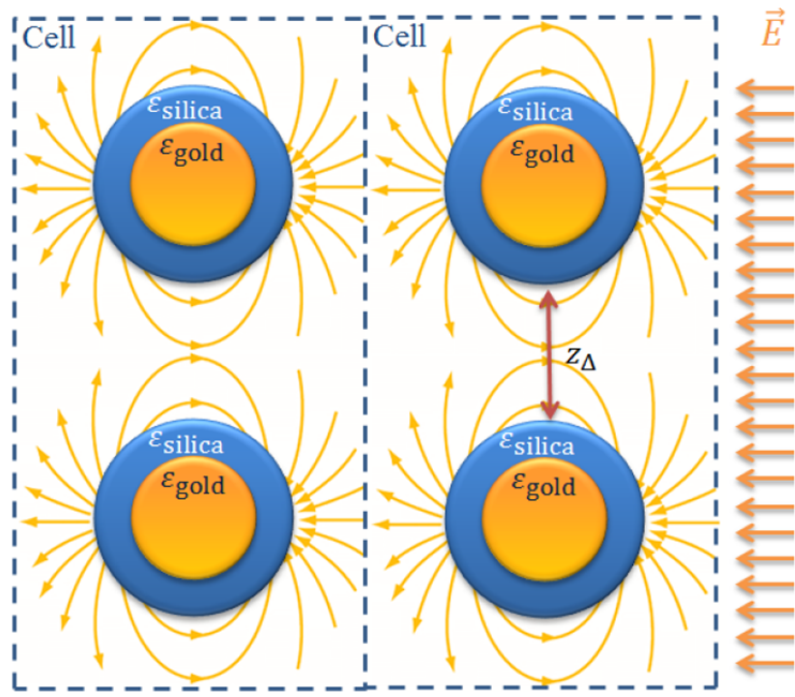

Fig. 4. Cells geometries of two NPM separated by a distance $z_{\Delta}$. 
In this model, each periodic cell possesses two nanoparticles and the cell walls are far away from the particles enough so that there is no interaction between the cells, but only between the two NPM of the cell. From this point view, we are interested in the case where the distance $z_{\Delta}$ is relatively small, allowing the interaction between the fields scattered by the particles. For this case of two coupled nanoparticles, we used the Comsol Multiphysics software, which possesses the RF module with the Frequency Domain subgroup for frequency electromagnetic studies. The geometry of the problem was defined in a three-dimensional model, where two core-shell particles of internal radius $r_{1}$, effective radius $r_{2}$ and thickness of the silica layer $\Delta$ were arranged laterally at a distance $z_{\Delta}$ between them (Fig. 5).

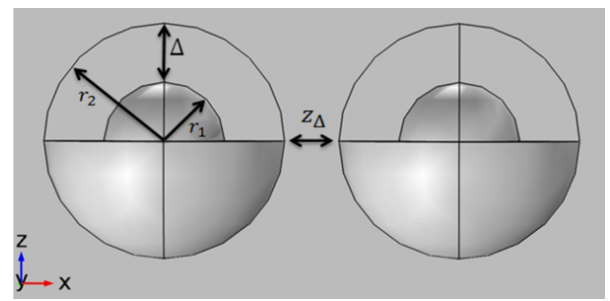

Fig. 5. Geometry of two coupled nanoparticles in FEM modeling.

The system mesh was defined according to the Physics Controlled Mesh function, where the software considers the refractive indices of the materials to obtain a model of the distribution of the electromagnetic field in the structure. The field type, Scattered Field, and Linearly Polarized Plane Wave, with data defined for normalized amplitude at $1 \mathrm{~V} / \mathrm{m}$, with wavelength $\lambda=632.8 \mathrm{~nm}$, were defined in the "The Electromagnetic Waves, Frequency Domain Interface". This source is equivalent to an example of laser equipment generally experiments. The domain of the model has a prismatic geometry, where the upper and lower faces were used Scattering Boundary Condition, which simulates the propagation of the polarized field in the direction $\widehat{a}_{x}$, and in the lateral faces were used Periodic Boundary Conditions, which simulate a periodic array of nanoparticles.

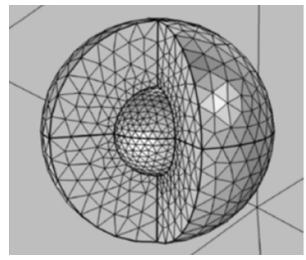

Fig. 6. Mesh discretization used in the Finite Element Method.

\section{RESUlts AND Discussions}

With the aid of the theoretical and experimental data available in literature [12], it was analyzed the parameters of scattering, absorption, extinction, absorbance and the electric field for spherical particles with different thickness of the shell.

The study was carried out using particles where the core radius ranges from $5 \mathrm{~nm}$ to $50 \mathrm{~nm}$, and cover layer with thickness ranging from $0 \mathrm{~nm}$ to $10 \mathrm{~nm}$, interacting with an electromagnetic wave of amplitude $E_{0}$ in aqueous medium (ethanol). The results were obtained in the wavelength range of $450 \mathrm{~nm}$ to $750 \mathrm{~nm}$, and the electric field distributions were calculated for wavelength $632.8 \mathrm{~nm}$, which is an example value generally used in practical experiments. We present some results of electric field distributions between two particles, in different arrangements, obtained by the FEM. We then analyze the convergence characteristics of the quasi-static method.

\section{A. Cross-Section Efficiency Analysis}

Fig. 7 shows the results of the normalized cross-sections of (29)-(30) of four core particles with radius of $5 \mathrm{~nm}, 15 \mathrm{~nm}$, $35 \mathrm{~nm}$ and $50 \mathrm{~nm}$, and layer thickness of $0 \mathrm{~nm}, 5 \mathrm{~nm}$ and $15 \mathrm{~nm}$.

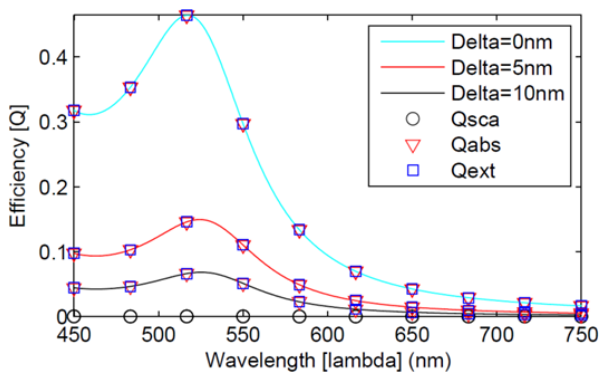

(a)

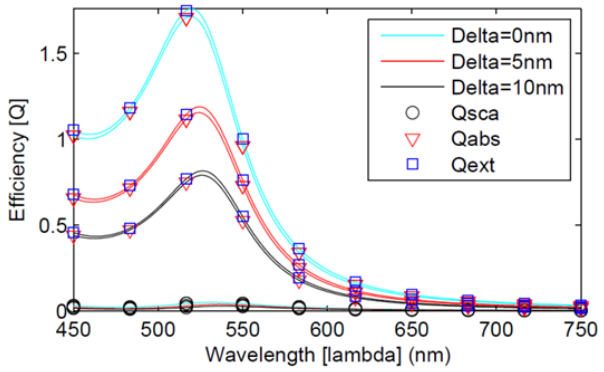

(b)

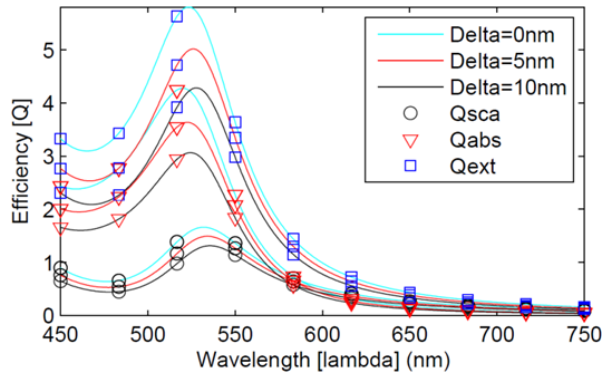

(c)

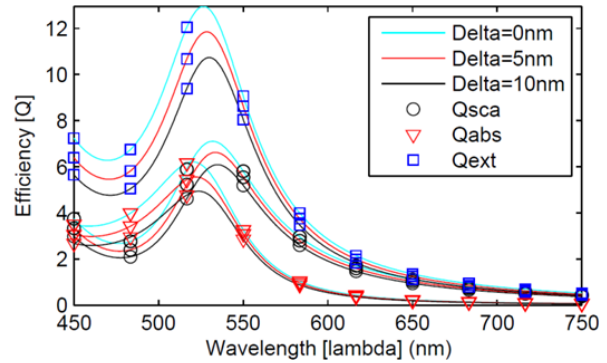

(d)

Fig. 7. Efficiency of scattering $\left(Q_{s c a}\right)$, absorption $\left(Q_{a b s}\right)$ and extinction $\left(Q_{\text {ext }}\right)$ of particles with (a) $r_{1}=5 \mathrm{~nm}$, (b) $r_{1}=15 \mathrm{~nm}$, (c) $r_{1}=35 \mathrm{~nm}$, (d) $r_{1}=50 \mathrm{~nm}$. 
It is verified that with the increase of the thickness of the dielectric layer there is a decrease in the cross-sections parameters of scattering and absorption. This is due to the increase in the geometric cross section area $S$ of (29)-(30) of the particle. This effect is more intense in the small particles, since the metallic core of the particle is the greater responsible for the dissipation of energy, and, for larger particles, the presence of a thin layer becomes less significant.

For smaller particles, the total extinction efficiency is caused predominantly by the absorbing characteristic, since the scattering is minimal. However, with the increase of the NPM is verified a greater contribution of the scattered energy. And finally, a red shift of the resonance point of extinction efficiency is observed for particles with larger layer thickness. This shows that the dielectric layer also modifies the resonance of the particles [24].

\section{B. Absorbance}

In this section, we are interested in the result of the interaction between a light beam propagating in the direction $\widehat{a}_{z}$ through a set of NPM (Fig. 4). Considering that the particles are at a great distance from each other and that the individual scattering is independent and does not interact with other particles, we can approximate the Absorbance for two samples containing NPM of 20nm,one without shell and the other with shell of $10 \mathrm{~nm}$, analytically from the extinction efficiency and compare with experimental results defined in [12]. The errors between the analytical model and the experimental data were calculated using (33).

$$
\operatorname{error}(\%)=\left(\frac{A b_{\text {real }}-A b_{\text {calculated }}}{A b_{\text {real }}}\right) \times 100 \%
$$

It was found that the spectral position of the resonance in the result of Fig. 8.a is faithful to that observed in the extinction efficiency for the particles without shell. This characteristic effect is caused by the LSPR (Localized Surface Plasmon Resonance) phenomenon, which in gold nanoparticles is centered near the wavelength of $520 \mathrm{~nm}$, and the characteristic behavior for smaller length values correspond to the absorption performed by electrons passing from the valence band to the conduction band. For the adopted model it is not useful to apply Fröhlich's condition to estimate the resonance band of the particle, since it would only be applicable if the imaginary component of the Drude model was very small, indeed close to zero in the frequency at which $\varepsilon\left(\omega_{R}\right)=-2 \varepsilon_{m}\left(\omega_{R}\right)$.The result in Fig. 8c shows good agreement with experimental data and a considerable shift of the resonance point to $529 \mathrm{~nm}$, caused by the presence of the shell. The errors for both cases, without and with shell, respectively, are shown in Fig.8b and Fig.8d.

\section{Electromagnetic Field Distributions}

In Section II we defined the expressions of the electric and magnetic fields resulting from the interaction between a core-shell type particle and a uniform field. The magnitude of electric field (Fig. 9) and magnetic field (Fig. 10), in $\lambda=632.8 \mathrm{~nm}$, were obtained for particles with core radius

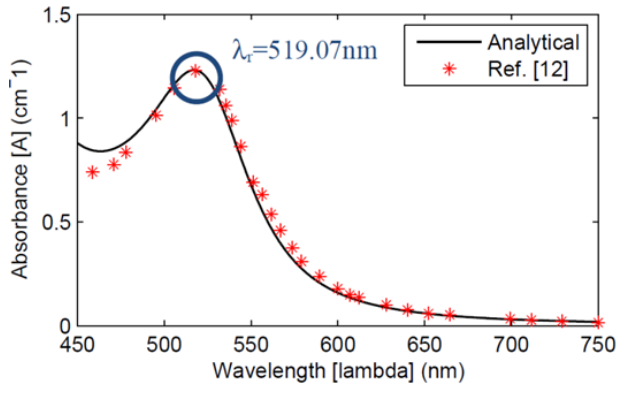

(a)

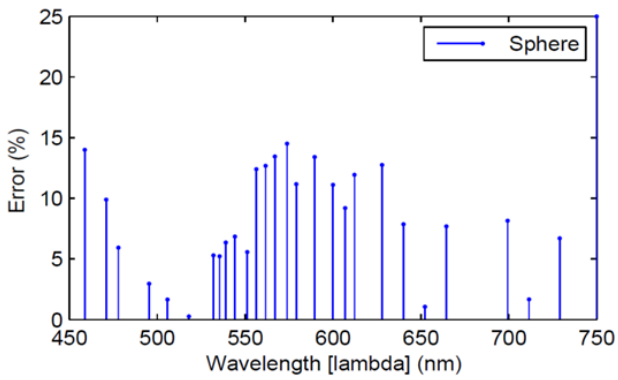

(b)

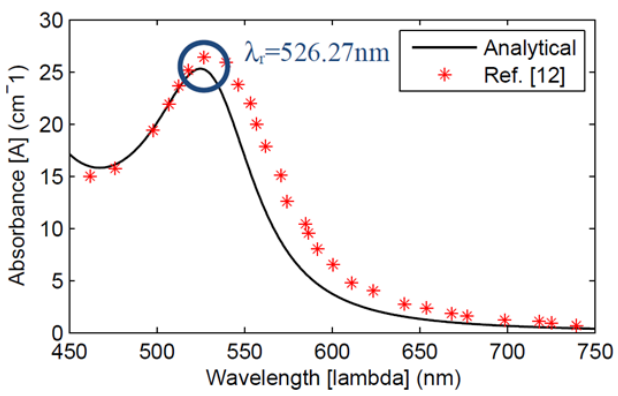

(c)

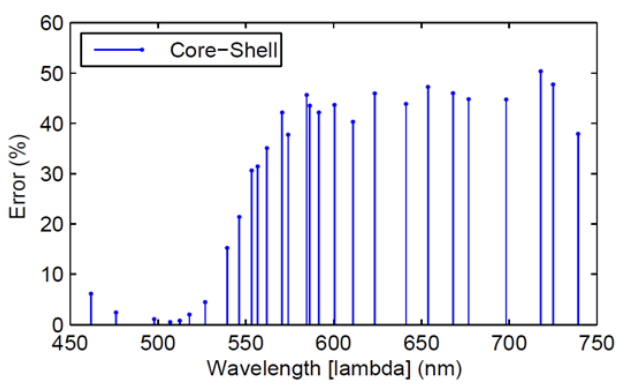

(d)

Fig. 8. Particle Absorbance of diameter 20nm: (a) Magnitude, (b) Error; Particle Absorbance core-shell of internal diameter $20 \mathrm{~nm}$ and shell of thickness $\Delta=10 \mathrm{~nm}$ : (a) Magnitude, (b) Error.

$a=5 \mathrm{~nm}, 15 \mathrm{~nm}, 35 \mathrm{~nm}$ and $50 \mathrm{~nm}$, with dielectric shell of thickness $\Delta=0 \mathrm{~nm}, 5 \mathrm{~nm}$ and $10 \mathrm{~nm}$, interacting with a uniform field with unit amplitude. Due the azimuthal symmetry of the problem, we can plot the fields in two-dimensional form, in the spatial variables $x$ and $z$. Such as, the color bars of the electric field module graphs were normalized to vary in the range of 0 to $4 \mathrm{~V} / \mathrm{m}$ making the visualization better, Magnetic field module plots have their color bars normalized to vary from 0 to $4 \mathrm{~mA} / \mathrm{m}$.

In Fig. 9 two characteristics are perceptible, the fundamental internal mode at the boundary where the particle is very small, which corresponds to the uniform internal electric field, and 

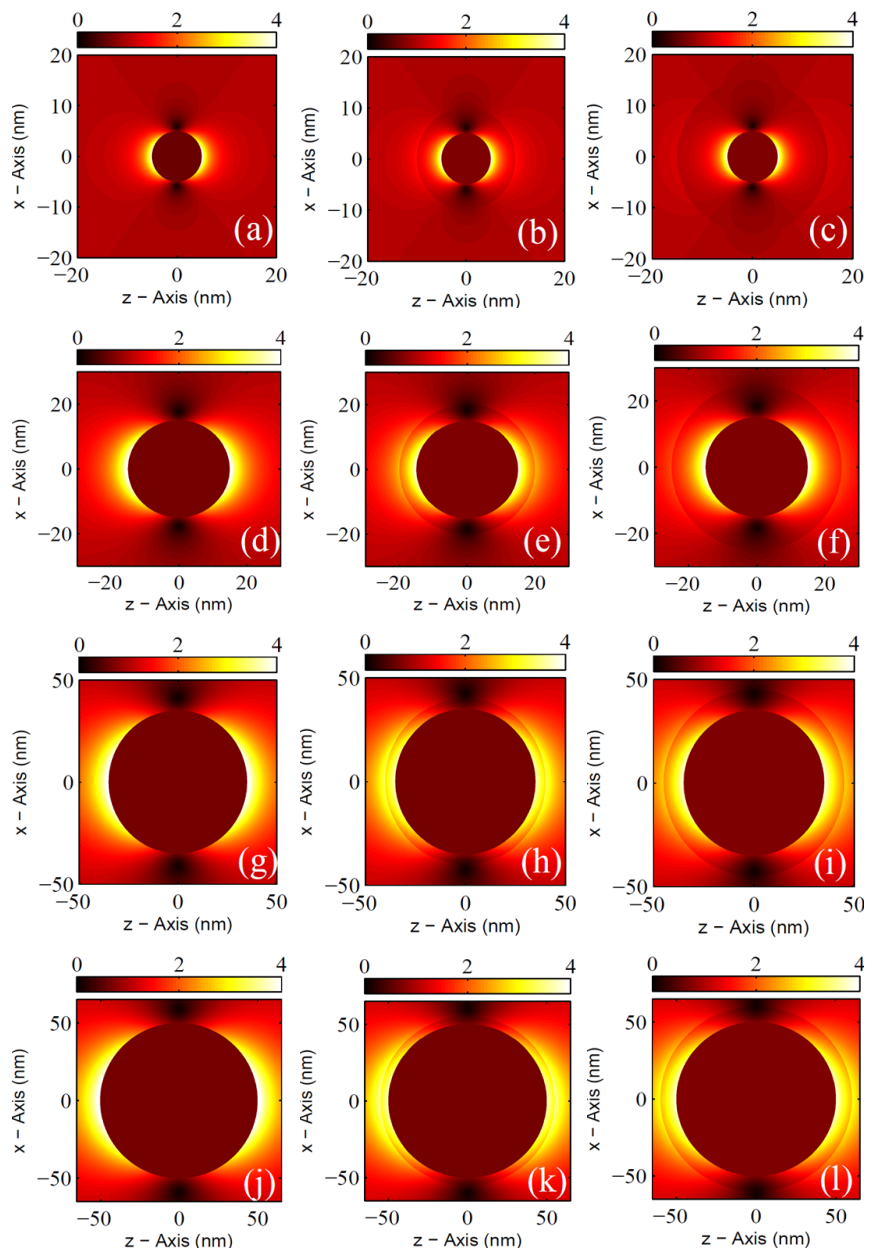

Fig. 9. Distributions of electric field module for particles with radius: (a) $r_{1}=5 \mathrm{~nm}, \Delta=0 \mathrm{~nm}$, (b) $r_{1}=5 \mathrm{~nm}, \Delta=5 \mathrm{~nm}$ (c) $r_{1}=5 \mathrm{~nm}, \Delta=10 \mathrm{~nm}$, (d) $r_{1}=15 \mathrm{~nm}, \Delta=0 \mathrm{~nm}$, (e) $r_{1}=15 \mathrm{~nm}, \Delta=5 \mathrm{~nm}$ (f) $r_{1}=15 \mathrm{~nm}$, $\Delta=10 \mathrm{~nm},(\mathrm{~g}) r_{1}=35 \mathrm{~nm}, \Delta=0 \mathrm{~nm},(\mathrm{~h}) r_{1}=35 \mathrm{~nm}, \Delta=5 \mathrm{~nm}$ (i) $r_{1}=$ $35 \mathrm{~nm}, \Delta=10 \mathrm{~nm}$, (j) $r_{1}=50 \mathrm{~nm}, \Delta=0 \mathrm{~nm}$, (k) $r_{1}=50 \mathrm{~nm}, \Delta=5 \mathrm{~nm}$ (l) $r_{1}=50 \mathrm{~nm}, \Delta=10 \mathrm{~nm}$.

the uniform oscillation of the electrons throughout the NPM volume, characterizing the dipolar oscillation of internal and external dipole moments.

For the adopted wavelength $(\lambda=632.8 \mathrm{~nm})$, it is verified that the internal field of the particle is very weak, in fact, smaller than the incident field. This phenomenon indicates that the metal for this frequency begins to gain dielectric properties, allowing the propagation of a weak field inside. With the increase of the particle radius there is a slight increase in the electric field intensity. It is also verified that the dipole effect on the surface of the silica layer has reduced amplitude in relation to the internal; this is because the permittivity of the medium and the silica layer are close, and in this way the field tends to maintain continuity at the interface between shell and medium.

We can note that the induced magnetic dipole have very weak amplitude, in fact, it is in agreement with the established in the theory for the quasi-static regime $[9,15]$. Note also that the dipole is induced from the core and presents continuity at the interface between the dielectric and the non-magnetic
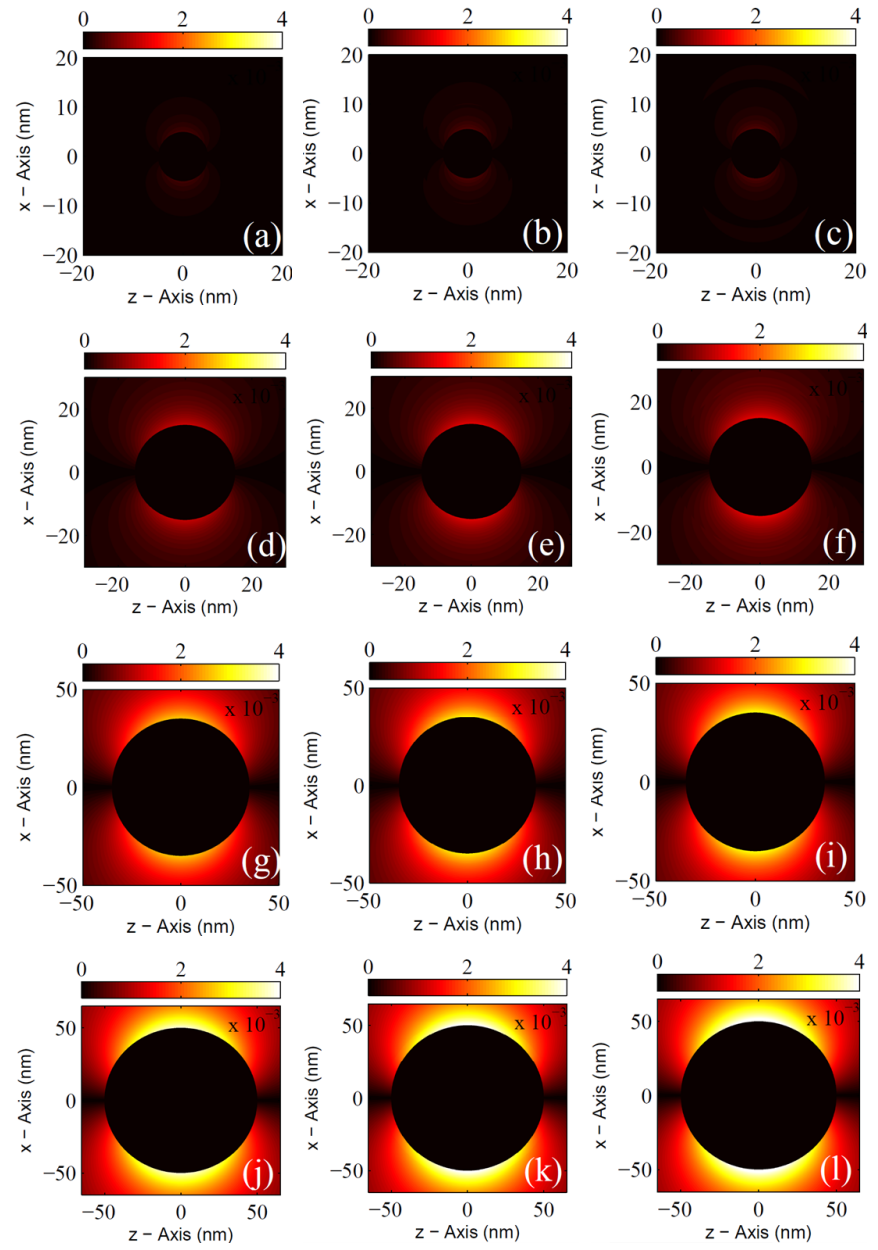

Fig. 10. Distributions of magnet field module for particles with radius: (a) $r_{1}=5 \mathrm{~nm}, \Delta=0 \mathrm{~nm}$, (b) $r_{1}=5 \mathrm{~nm}, \Delta=5 \mathrm{~nm}$ (c) $r_{1}=5 \mathrm{~nm}, \Delta=10 \mathrm{~nm}$, (d) $r_{1}=15 \mathrm{~nm}, \Delta=0 \mathrm{~nm}$, (e) $r_{1}=15 \mathrm{~nm}, \Delta=5 \mathrm{~nm}$ (f) $r_{1}=15 \mathrm{~nm}$, $\Delta=10 \mathrm{~nm}$, (g) $r_{1}=35 \mathrm{~nm}, \Delta=0 \mathrm{~nm}$, (h) $r_{1}=35 \mathrm{~nm}, \Delta=5 \mathrm{~nm}$ (i) $r_{1}=$ $35 \mathrm{~nm}, \Delta=10 \mathrm{~nm}$, (j) $r_{1}=50 \mathrm{~nm}, \Delta=0 \mathrm{~nm}$, (k) $r_{1}=50 \mathrm{~nm}, \Delta=5 \mathrm{~nm}$ (l) $r_{1}=50 \mathrm{~nm}, \Delta=10 \mathrm{~nm}$.

aqueous medium $\left(\mu_{m}=\mu_{0}\right)$. With these conclusions, we will focus in analyzing the electric field in later topics.

From (19), we plot the electric potential in Fig. 11, with reference in the origin, for three particles of radius $r_{1}=5 \mathrm{~nm}$ and dielectric shells $\Delta=0 \mathrm{~nm}, 5 \mathrm{~nm}$ and $10 \mathrm{~nm}$, in order to observe the changes and interactions.
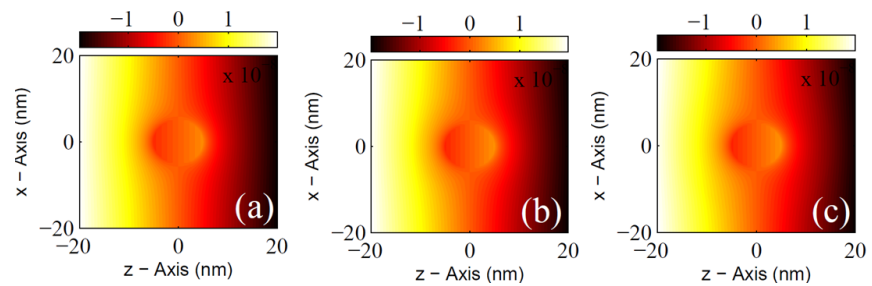

Fig. 11. Electric potential distributions $V(r, \theta)$ : (a) $r_{1}=5 \mathrm{~nm}, \Delta=0 \mathrm{~nm}$, (b) $r_{1}=5 \mathrm{~nm}, \Delta=5 \mathrm{~nm}$ (c) $r_{1}=5 \mathrm{~nm}, \Delta=10 \mathrm{~nm}$.

It is known that in a uniform field, the electric potential varies almost linearly in the $z$ direction, which is why we observe the behavior of Fig. 11, where the electric potential decreases in the direction of the electric field and rises in 
the opposite direction, characterizing the phase of the field incident. However, we find that internally to the particle core, there is an inversion of the field phase, which increases in the direction of the incident electric field. We can verify this phenomenon by generating graphs of the electric field phase (Fig. 12) for the three particles previously used.
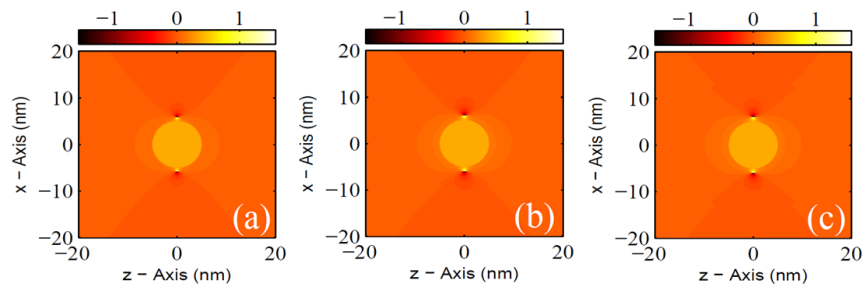

Fig. 12. Electric field phase: (a) $r_{1}=5 \mathrm{~nm}, \Delta=0 \mathrm{~nm}$, (b) $r_{1}=5 \mathrm{~nm}$, $\Delta=5 \mathrm{~nm}$ (c) $r_{1}=5 \mathrm{~nm}, \Delta=10 \mathrm{~nm}$.

Finally, we present in Fig. 13 the three-dimensional graphs of electric and magnetic fields to demonstrate the dipolar characteristic of the core-shell particles in a volumetric region.

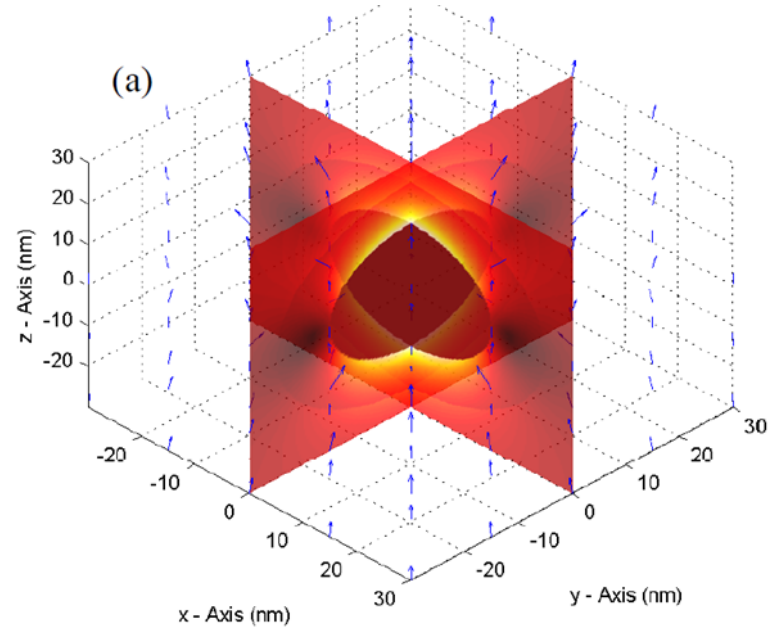

(b)

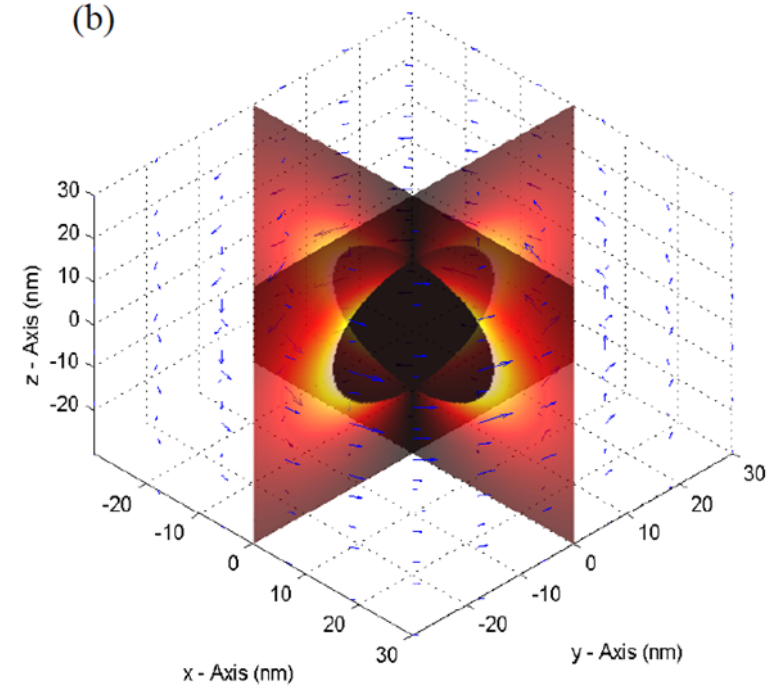

Fig. 13. . Three dimensional distributions of the electromagnetic fields of a core-shell nanoparticle with $r_{1}=15 \mathrm{~nm}$ and $\Delta=10 \mathrm{~nm}$ : (a) Electric; (b) Magnetic.
The internal field of the gold sphere has a phase delay in relation to the incident field, this is the main characteristic of the plasmon effect inside the particle, caused by the real negative part of the dielectric function of metal. Note that in regions distant from the particle, the field has zero phase. we can verify a rotational characteristic of the magnetic field around the NPM, expected behavior.

\section{Maximum Electric Field Intensity at Core and Dielectric Layers}

Results were generated for four particles of internal radius $r_{1}=5 \mathrm{~nm}, 15 \mathrm{~nm}, 35 \mathrm{~nm}$ and $50 \mathrm{~nm}$ in the verification of the maximum electric field on the surfaces of the gold sphere and the silica layer. These particles had the thickness of their layers varied from 0 to $10 \mathrm{~nm}$, and were subjected to an incident field with wavelength variation from $450 \mathrm{~nm}$ to $750 \mathrm{~nm}$.
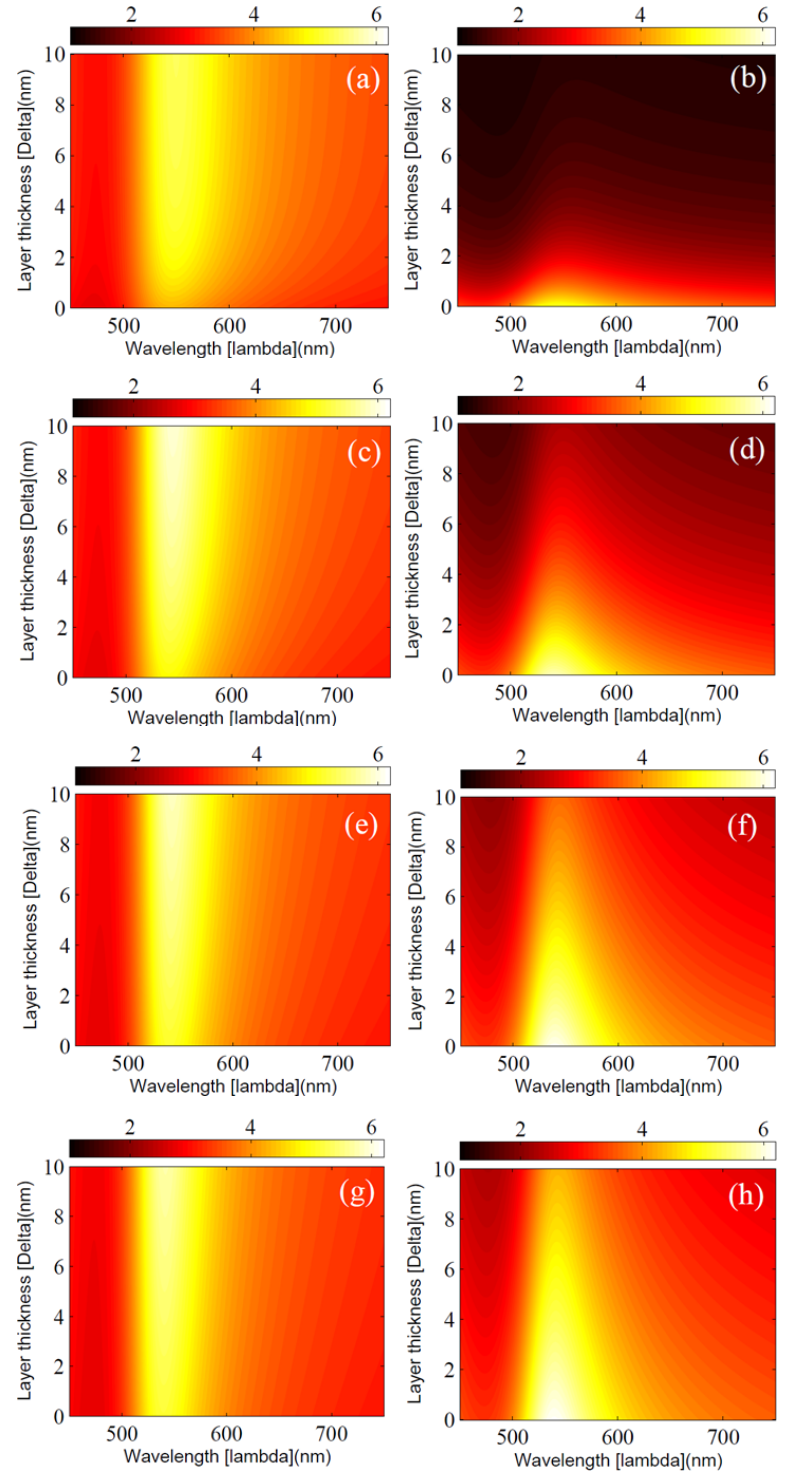

Fig. 14. Variation of the electric field intensity in function of wavelength $(\lambda)$, thickness of dielectric layer $(\Delta)$ and radius of core $\left(r_{1}\right)$ at the points: (a) $r=$ $r_{1}=5 \mathrm{~nm}$ (b) $r=r_{2}=5 \mathrm{~nm}+\Delta$, (c) $r=r_{1}=15 \mathrm{~nm}$, (d) $r=r_{2}=15 \mathrm{~nm}$ $+\Delta$, (e) $r=r_{1}=35 \mathrm{~nm}$ (f) $r=r_{2}=35 \mathrm{~nm}+\Delta,(\mathrm{g}) r=r_{1}=50 \mathrm{~nm}$, (h) $r=r_{2}=50 \mathrm{~nm}+\Delta$, with $\theta=\phi=0$. 
The graphs were generated at $r=r_{1}$, on the surface of the gold sphere, and $r=r_{2}$ on the surface of the silica layer, with $\theta=\phi=0$ (Fig. 14), taking azimuth symmetry into account. The color bars of graphs were normalized to vary in the range of 1 to $6.18 \mathrm{~V} / \mathrm{m}$ making the visualization better.

As previously noted, for particles with the inner radius very small relative to the shell, the field in the shell region becomes less intense as its thickness is increased, but in contrast the core field is amplified. This effect is extended to larger particles. We also observe that the resonances in these figures are in agreement to those $Q_{a b s}$ presented in Fig. 7, because these near field produce the absorptions of the particles.

\section{E. Electric Field Distributions of Two Coupled NPMs}

Using the Comsol software, the electric field distributions of two coupled core-shell nanoparticles were calculated in functions of the distance between them. The particles possess internal diameter of $10 \mathrm{~nm}$ and shell with thickness of $10 \mathrm{~nm}$. The particles were positioned parallel to each other by varying the gap $\left(z_{\Delta}\right)$ between them in $5 \mathrm{~nm}$ and $20 \mathrm{~nm}$, with the amplitude field $E_{0}=1 \mathrm{~V} / \mathrm{m}$ in the directions of $\mathrm{z}$ and $\mathrm{x}$. The results are shown in Fig. 15.
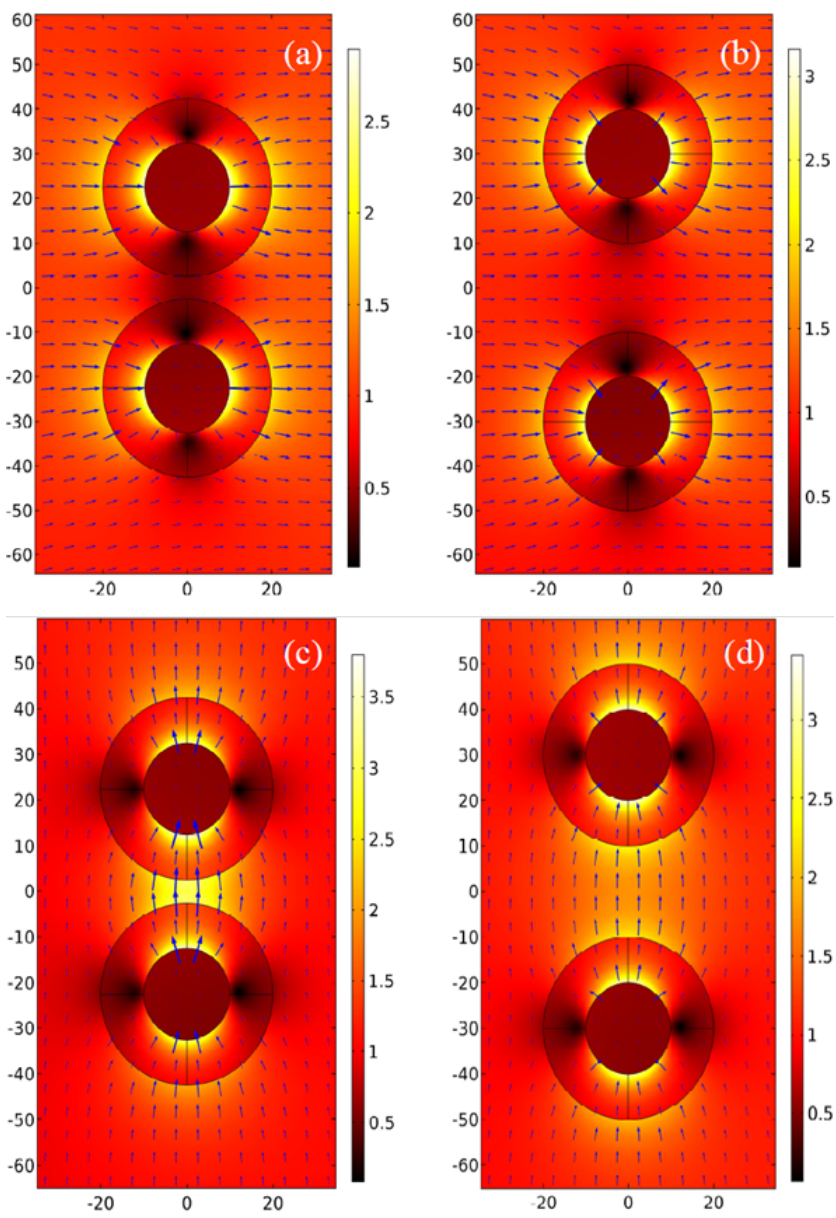

Fig. 15. Distributions of electric field module for two coupled core-shell particle for different distances $z_{\Delta}$ and polarization of the incident field: (a) $z_{\Delta}=5 \mathrm{~nm}, \widehat{u}=\widehat{a}_{x}$, (b) $z_{\Delta}=20 \mathrm{~nm}, \widehat{u}=\widehat{a}_{x}$, (c) $z_{\Delta}=5 \mathrm{~nm}, \widehat{u}=\widehat{a}_{z}$, (d) $z_{\Delta}=20 \mathrm{~nm}, \widehat{u}=\widehat{a}_{z}$.
In Figs. 15a and $\mathrm{b}$ the incident field is perpendicular to the axis of the two particles, and Figs. $15 \mathrm{c}$ and d, the incident electric field is parallel to the axis of the particles.

We observe that the electric field between the particles is higher for the cases of Figs. 15c and d than the cases of Figs. $15 \mathrm{a}$ and $\mathrm{b}$. This is due because there is strong dipolar coupling between the particles in Figs. $\mathrm{c}$ and d, as can be noted by the electric field distributions, and this coupling is increased for smaller $z_{\Delta}$.

\section{F. Validity of the Quasi- Static Model}

The method used here has a convergence region as a function of particle size, and as defined above, it is based on the assumption that the nanoparticle has a radius much smaller than the wavelength of the incident field, $r \ll \lambda$, however, only this statement is not able to define a region of convergence of the method.

The Mie scattering is the exact solution for the interaction between a spherical particle of radius $R$ and an electromagnetic wave for all electrical size $R / \lambda$ [15]. In this model, the field resulting from the interaction is a superposition of normal modes, called spherical harmonics, describing a multipolar expansion. The Mie model can be reduced to a dipole behavior when the approximation are used in the Rayleigh model, $r \ll \lambda$, which for the model of Mie represents $x \ll 1$, where:

$$
x=2 \pi \frac{R}{\lambda} \sqrt{\varepsilon_{m}}
$$

Fig. 16 shows a plot of (34) in function of wavelength lambda and radius $R$.

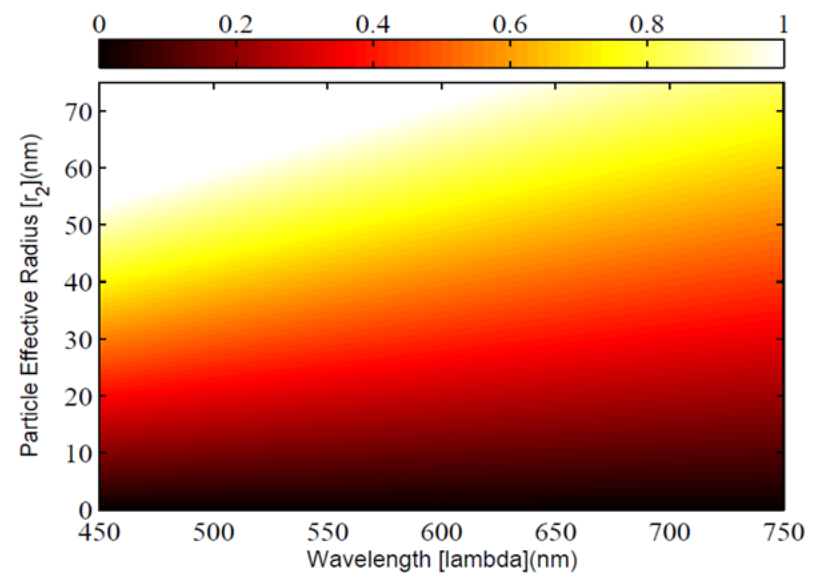

Fig. 16. Plot of the convergence parameter $x(R, \lambda)$ of (33). The convergence of the method is better when $x \ll 1$.

From Fig. 16, we see that the convergence of the method decreases with the increase of the frequency and radius of the NPM, that is, when $x$ approaches values close to 1 . This relation provides a numerical range of validity for the almost static model. It is important to remember that the dielectric function used also has a convergence region, and even in the general Mie model, the modeling tends to diverge outside the convergence region of the Lorentz Drude model. 


\section{CONCLUSiON}

This work presented a theoretical study of the resonant response of gold nanospheres covered by dielectric layers (core-shell). In the case of isolated particles, the quasi-static approximation was used for analysis, i.e., Rayleigh scattering, and for the case of coupled particles the finite element method was used for numerical analysis. It was observed that for greater thickness of the dielectric layer, keeping the internal radius constant, the resonant wavelength increases. This result shows that, in precision experiments, this dielectric layer should be considered in the analysis to avoid errors of calculations of the resonant response. It was verified that the magnet field has low intensity, which is given by the intrinsic impedance of the medium, defined by the constitutive parameters of the materials. It was also observed that the scattering of the particle increases with the size of the internal radius, and that the quasi-static model is suitable for particles with mean radius approximately smaller than $50 \mathrm{~nm}$, where for larger particles the Mie scattering model should be used. In the case of coupled particles, preliminary results of the interaction between core-shell particles were presented for different polarizations of the incident field, however, a more detailed analysis on the extinction efficiency becomes necessary, and it is proposed as future works. Another proposal may be the using of the polarizabilities presented here to obtain an effective permittivity of an array of core-shell particles through the Clausius-Mossotti model.

\section{ACKNOWLEDGMENT}

The authors thank the members of the Laboratory of Electromagnetism (LEMAG-TUC-UFPA).

\section{REFERENCES}

[1] X. Huang, R. O'Connor, E. A. Kwizera, "Gold Nanoparticle Based Platforms for Circulating Cancer Marker Detection". Nanotheranostics 2017.

[2] J. Guo, K. Rahme, Y. He, L-L. Li, J.D. Holmes, C.M. O'Driscoll, "Gold nanoparticles enlighten the future of cancer theranostics". International Journal of Nanomedicine. 2017

[3] R. C. Santos, A. F. S. Cruz, J. S. Costa, K. Q. Costa. "Análise do Efeito da Distância entre Nanopartículas de Ouro na Resposta de Sensores Plasmônicos.” In: SBrT 2017, 2017, São Pedro - SP. XXXV Simpósio Brasileiro de Telecomunicações e Processamento de Sinais, 2017.

[4] Y. Q. He, S. P. Liu, L. Kong, and Z. F. Liu, "A study on the sizes and concentrations of gold nanoparticles by spectra of absorption, resonance rayleigh scattering and resonance non-linear scattering," Spectrochimica Acta, Part A, no. 61, pp. 2861-2866, 2005.

[5] M. K. Pereira, "Ressonância de plasmon de superfície localizado e espalhamento raman em soluções coloidais de ouro," M.S. thesis, Instituto de Física - UFRGS, Porto Alegre, RS, Brazil, 2009.

[6] J. P. Wilcoxon and B. L. Abrams, "Synthesis, structure and properties of metal nanoclusters," Chemical Society Reviews, Vol. 35, p. 1162, 2006.

[7] K. Q. Costa and V. Dmitriev, "Simple and efficient computational method to analyze cylindrical plasmonic nanoantennas," in $J$. of $A n-$ tennas and Prop., vol. 2014, ID. 675036, pp. 1-8, 2014.

[8] S. A. Maier, "Localized surface plasmons," in Plasmonics: Fundamentals and Applications, $1^{\text {st }}$ ed. New York, NY, USA: Springer, 2007, pp. 65-87.

[9] X. Liu, M. Atwater, J. Wang, and Q. Huo, "Extinction coefficient of gold nanoparticles with different sizes and different capping ligands," Colloids and Surfaces B - Biointerfaces no. 58, pp. 3-7, 2007.

[10] G. A. Rance, D. H. Marsh, and A. N. Khlobystov, "Extinction coefficient analysis of small alkanethiolate-stabilised gold nanoparticles," Chemical Physics Letters, no. 460, pp. 230-236, 2008.
[11] COMSOL Multiphysics 5.2, Inc. Available: https://www.comsol.com. Accessed in: 19/01/2016.

[12] NanoComposix. Available: https://nanocomposix.com/. Accessed in: 07/03/2016.

[13] A. F. S. Cruz, R. C. Santos, P. F. Ferraz, J. S. Costa and K. Q. Costa, "Estudo analítico do espalhamento de rayleigh em nanopartículas de ouro do tipo core-shell," in MOMAG: $12^{\circ}$ CBMAG, $17^{\circ}$ SBMO and LAWOFS, Porto Alegre, RS, Brazil, 2016, pp. 1-6.

[14] G. R. Fowles, "Optics of solids," in Introduction to Modern Optics, $2^{\text {nd }}$ ed. New York, NY, USA: Rinehart and Winston, 1968, pp. 151-154.

[15] C. F. Bohren and D. R. Huffman, "Particles small compared with the wavelength," in Absorption and Scattering of Light by Small Particles, $1^{\text {st }}$ ed. New York, NY, USA: Wiley, 1983, pp. 130-154.

[16] RefractiveIndex.INFO, "Refractivity Index of Fused Silica." Available: http://refractiveindex.info/?shelf=main\&book=SiO2\&page=Malitson. Accessed in: 13/02/2016.

[17] K. Q. Costa, J. S. Costa, V. Dmitriev, T. D. Rosso, O. Pandoli, and R. Q. Aucelio, "Analysis of surface plasmon resonance sensor coupled to periodic array of gold nanoparticles," in IMOC: SBMO/IEEE MTT-S International, Porto de Galinhas, PE, Brazil, 2015, pp. 1-5.

[18] A. Jeffrey, "Series solutions of differential equations, special functions, and sturm-liouville equations," in Advanced Engineering Mathematics, $1^{\text {st }}$ ed. San Diego, CA, USA: Harcourt, 2001, pp. 443-526.

[19] G. B. Arfken and H. J. Weber, "Legendre functions," in Mathematical Methods For Physicists, $6^{\text {th }}$ ed. San Diego, CA, USA: Elsevier, 2005, pp. 741-816

[20] M. N. O. Sadiku, "Analytical Methods," in Numerical Techniques in Electromagnetics, $2^{\text {nd }}$ ed. New York, NY, USA: CRC, 2001, pp. 66-78.

[21] J. A. Stratton, "The electrostatic field," in Electromagnetic Theory, $1^{\text {st }}$ ed. New York, NY, USA: McGraw-Hill, 1941, pp. 197-205.

[22] J. D. Jackson, "Boundary-Value Problems in Electrostatics: II," in Classical Electrodynamics, $3^{\text {rd }}$ ed. Hoboken, CA, USA: John Wiley \& Sons, 1999, pp. 95-135.

[23] A. F. S. Cruz, P. F. Ferraz, R. C. Santos, K. Q. Costa, "Análise do campo elétrico em nanoesfera com camada de grafeno," in XI ENEEEL, Natal, RN, Brazil, 2016, pp. 1-12.

[24] A. F. S. Cruz, R. C. Santos, J. S. Costa and K. Q. Costa, "Eficiência de extinção em nanopartículas metálicas do tipo core-shell isoladas e acopladas," in XXXIV SBrT, Santarém, PA, Brazil, 2016, pp. 1-5.

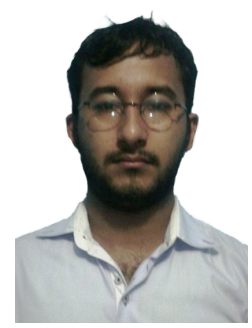

André Felipe S. da Cruz holds a degree in Electrical Engineering (2016) and holds a Master's degree in Electrical Engineering from the Federal University of Pará. He has experience in Electronics.

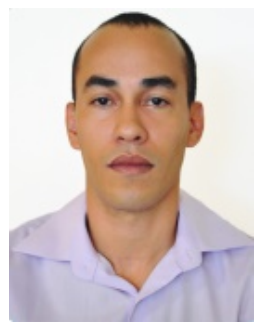

Karlo Q. da Costa has a degree (2000), a master's degree (2002), a doctorate (2006) and a postdoctoral degree (2011) in Electrical Engineering from the Federal University of Pará. He is currently Associate Professor at the Federal University of Pará. Electrical Engineering, with emphasis in Electromagnetic Theory, Microwave, Wave Propagation, Antennas, acting mainly in the following subjects: method of the moments, electromagnetic theory, plasmonics, nanoantenas, nanoelectronic and nanophotonics. 